\title{
The Optimization of Wideband Cyclostationary Feature Detector with Receiver Constraints
}

\author{
Ikedieze Gabriel Anyim, John Chiverton, Misha Filip and Abdulkarim Tawfik. \\ University of Portsmouth, United Kingdom. \\ Email: gabriel.anyim@port.ac.uk
}

\begin{abstract}
Cognitive radio system is a context-aware technology in communications. Spectrum awareness is an important function in the design of cognitive radio systems. It senses the presence or absence of primary users in the spectrum and declares the unoccupied channels for secondary users. Cyclostationary Feature Detection is about the detection of signals based on their features such as cyclic frequencies, symbol rates, carrier frequencies and modulation types. Detection can occur at very low signal to noise ratios.However performance degrading constraints such as cyclic and sampling clock offsets can occur at the receiver through the local oscillator frequency offsets, Doppler effects and jitter. We propose a multi-slot cyclostationary feature detector that reduces the effects of these constraints by optimizing for the number and size of each slot and fast Fourier transform. These slots and fast Fourier transforms are used to show the reduction of these offsets and the detection performance is compared for different scenarios with and without offsets.
\end{abstract}

Keywords - cognitive radio, spectrum sensing, cyclostationary, cyclic autocorrelation function, spectral correlation function.

\section{Introduction}

The radio spectrum is a resource that has a natural limited availability. The need therefore arises for its efficient utilization in order to provide users with different services at higher data rates. As a result of increasing demand for the spectrum largely due to the fast expanding market of wireless communication subscribers and also high data applications, these radio frequencies are becoming scarce. Additionally, spectrum segmentation and dedicated frequency allocation of the standardized wireless systems are contributing to the scarcity. Efficient spectrum usage is now a critical issue due to these demands. On the other hand, various spectrum usage measurements and monitoring campaigns across different parts of the world shows that a substantial amount of the wireless spectrum are under-utilized over a wide range of radio frequencies [1]. The Federal Communications Commission (FCC) of the United States have done survey measurements which indicated that several licensed frequency bands are unused up to ninety percent of the time [2]. Presently, wireless networks are regulated by fixed spectrum assignment policies, where the spectrum is regulated by governmental agencies such as the Office of Communications (OFCOM) in the United Kingdom (UK). Frequencies are allocated mostly on a long term basis for large or regional geographical regions. The diverse scarcity of communication spectrum has become one of the major issues for the development of new communication systems. In this context, Cognitive Radio (CR) can adapt to its surrounding communication environment and has emerged as a promising solution to address this spectrum scarcity by exploring spectral opportunities and deliver a more efficient utilization of the available spectral resources [3].

The CR concept can be divided into four main functional areas namely: (A) "Spectrum sensing" which determines the spectrum availability and the presence or absence of licensed primary users. (B) "Spectrum management" which predicts the duration that the spectrum holes (unused bandwidths) will be available for the secondary users before it is released again to the primary users [4]. (C) "Spectrum sharing" for allocating the spectrum holes among the secondary users according to demand. (D) "Spectrum mobility" which maintains hitless or error free (seamless) communication during frequency allocation between the primary and secondary users and thereby produce better spectrum transition [5]. This research is on Spectrum sensing of the CR.

Some researched spectrum awareness systems such as Energy Detection and Matched Filters are not capable of detecting signals at low signal to noise ratio (SNR)in order to provide dynamic and accurate information to the secondary users [6], [5] and [7]. On the other hand, Cyclostationary Feature Detector (CFD) detects signals at low level SNRs through the features calculated from modulated signals such as spectrum and cyclic frequencies. It can also be implemented in a wideband to reflect the real world communications environment. Some authors have looked at wideband cyclostationary feature detector as in [8] and [6] in different contexts using compressed measurements and Welch periodogram respectively . In this paper, we propose a multi-slot wideband cyclostationary feature detector statistical model that uses spectral correlation function (SCF) and window-based fast Fourier Transform (FFT) to reduce the effects of receiver constraints namely: sampling clock offset (SCO) and cyclic frequency offset (CFO). The rest of this paper is divided into 3 sections as follows: In section II, we looked at the conventional principles of spectral correlation function as applicable to CFD and showed by simulations the effects of sampling clock offset (SCO) and cyclic frequency offset (CFO) on the received signal. In section III, the proposed system model was introduced and analyzed highlighting the SCO and CFO. This was followed by more simulations in section IV to arrive at the required slot and FFT sizes and numbers required to reduce the effects of the offsets.

\section{Cyclostationary Feature Detection}

The fundamental principles used for feature detection along with the receiver constraints that affect the performance of 
the CFD will be discussed.

\subsection{Spectral Correlation Function}

The Autocorrelation function (AF) of a signal $x(t)$ as a function of time $t$ with period $T$ is the similarity of a function with itself at time lag $\tau$. A process is said to show cyclostationarity if its Autocorrelation function and mean are periodic. As discussed in [9] and [10], since the AF is periodic, it can be represented by Fourier series as:

$$
R_{x}\left(t+\frac{\tau}{2}, t-\frac{\tau}{2}\right)=\sum_{\alpha} R_{x}^{\alpha}(\tau) e^{j 2 \pi \alpha t},
$$

where $\alpha$ is the cyclic frequency at which the cyclostationary feature or second order periodicity of the modulated signal occurs. $R_{x}^{\alpha}(\tau)$ are the Fourier series coefficients and give the generalized Cyclic Auto-correlation Function (CAF) shown in (2),

$$
R_{x}^{\alpha}(\tau)=\frac{1}{T} \int_{-T / 2}^{T / 2} R_{x}\left(t+\frac{\tau}{2}, t-\frac{\tau}{2}\right) e^{-j 2 \pi \alpha t} \mathrm{~d} t .
$$

Also, it can be symmetrically expressed to reflect the nature of a modulated signal in real world communications as,

$$
R_{x}^{\alpha}(\tau)=\lim _{T \rightarrow \infty} \frac{1}{T} \int_{T} x\left(t+\frac{\tau}{2}\right) x\left(t-\frac{\tau}{2}\right) e^{-j 2 \pi \alpha t} \mathrm{~d} t
$$

where $T$ is the fundamental period and for a modulated signal it is a function of the symbol period and carrier frequency as in [9]. When $\alpha$ is zero, (3) gives the conventional Cyclic Autocorrelation Function (CAF) whose Fourier transform (FT) is the Spectral Correlation Function (SCF) for the observation time $T^{\prime}$ and expressed as,

$$
\begin{aligned}
S_{x}^{\alpha}(f)=\lim _{1 / T^{\prime} \rightarrow 0} \lim _{T \rightarrow \infty} \frac{1}{T^{\prime}} \int_{-T^{\prime} / 2}^{T^{\prime} / 2} & \frac{1}{T} X_{T}\left(t, f+\frac{\alpha}{2}\right) \\
& \times X_{T}^{*}\left(t, f-\frac{\alpha}{2}\right) \mathrm{d} t
\end{aligned}
$$

where $X_{T}(t, f)$ is the complex envelope of the narrow-band spectral component or short-time Fourier Transform of $x(t)$ with centre frequency $f$, period $T$ (FT length), bandwidth $1 / T$ (of the FT) and cyclic frequency $1 / T^{\prime}$. Further expressed as,

$$
X_{T}(t, f)=\int_{t-T / 2}^{t+T / 2} x(t) e^{-j 2 \pi f t} d t .
$$

Given $N^{\prime}$ number of samples, the SCF can be represented discretely with,

$$
S_{x}^{\alpha}(n, f)=\frac{1}{N^{\prime}} \sum_{n=0}^{N^{\prime}-1} \frac{1}{L} X_{L}\left(n, f+\frac{\alpha}{2}\right) X_{L}^{*}\left(n, f-\frac{\alpha}{2}\right)
$$

as discussed in [11] and [12], where $N^{\prime}$ is the sensing time, $X_{L}(l, f)$ is the $L$-point fast Fourier transform (FFT) around the $n^{t h}$ sample with $L$ as the discrete FFT length. We refer to (6) as the conventional discrete Spectral Correlation Function. Cyclostationary Feature Detector (CFD) uses either the CAF or SCF in time and frequency domains respectively to detect cyclic features of the received modulated signal such as cyclic and spectrum frequencies as discussed in [13], [12], [14] and [15].

\subsection{Effect of Cyclic Frequency and Sampling Clock Offsets on Spectral Correlation Function}

Cyclic frequency $\alpha$ is a function of both symbol rate $1 / T$ and carrier frequency multiples $2 f_{c}$, where $f_{c}$ is the carrier frequency and $T$ is the symbol period set by the clock at the transmitter [11] and [12]. Every clock produces some errors and in a local oscillator this results in frequency offsets as discussed in [15] and [11]. Also some uncertainties in the cyclic frequency will be introduced by Doppler shift. Cyclostationary Feature Detector (CFD) requires knowledge of the cyclic frequency and symbol rate to correctly detect the signals. Therefore, sub-optimal knowledge of cyclic frequency, symbol rate and carrier frequency results in cyclic frequency offset (CFO) at the receiver and this affects the detection performance of CFD and largely at increasing number of samples as discussed in [15] and [16]. Let the CFO be represented by $\Delta_{\alpha}$. Given that,

$$
\alpha^{\prime}=\alpha \times\left(1+\Delta_{\alpha}\right)
$$

where $\alpha^{\prime}$ and $\alpha$ are the ideal and actual cyclic frequencies (CF) at the Receiver and transmitter respectively. Some modulated signals have cyclic frequencies at both symbol rate and carrier frequency. The effect of CFO can be determined by substituting (7) for $\alpha$ in SCF (4). Some studies were carried out on the reduction of the impacts of $\mathrm{CFO}$ on the $\mathrm{CFD}$ using the Cyclic Autocorrelation Function (CAF) for analysis as discussed in [16]. The use of spectral correlation function (SCF) and fast Fourier transform (FFT) to reduce the effects of CFO and SCO has not been fully explored. Analysis in the frequency domain with FFT is less complex and computationally efficient as compared with in the time domain [17] and hence it is the adopted approach of this research to use SCF and FFT to reduce the effects of $\mathrm{CFO}$ and SCO. Sampling Clock Offset (SCO) is another condition that affects feature detection. It occurs from the frequency offset produced by oscillators and insufficient knowledge of the symbol rates at the Analogue to Digital (A/D) stage of the receiver. Sampling frequencies are produced by these oscillators, with $\mathrm{SCO} \delta$, and results in a drift in sampling times and this time-shift varies as the number of samples increases [16]; producing phase shifts and affecting the cyclic frequency. Fig. 1(a) shows the SCF of QPSK signal without any CFO while in Fig. 1(b), the cyclic frequency is being shifted in position as a result of the presence of CFO the possibility of correctly sensing the primary user's (PU) signal. SCO can be stated as in (8),

$$
T_{s}=(1+\delta) \times T_{s}^{\prime}
$$

where $T_{s}$ is the sampling period used at the receiver, $T_{s}^{\prime}$ is the actual sampling period with good knowledge of the symbol rate at the transmitter and $\delta$ is the SCO. In order to adequately represent a signal, the sampling rate $1 / T_{\text {symbol }}$ is in multiples of the symbol rate, where $T_{\text {symbol }}$ is the symbol period. The objective of the model is to remedy these effects so as to improve the possibility of detection through a statistical model using the SCF and FFT-based slot in a wideband scenario.

\section{System Model}

We propose a wideband multi-slot window-based FFT statistical and Test based CFD model that detects signals $x(t)$ by correlating in the frequency domain with the spectral correlation function (SCF) defined in (4) and (6). It covers the possibilities of reducing the effects of CFO and SCO. 

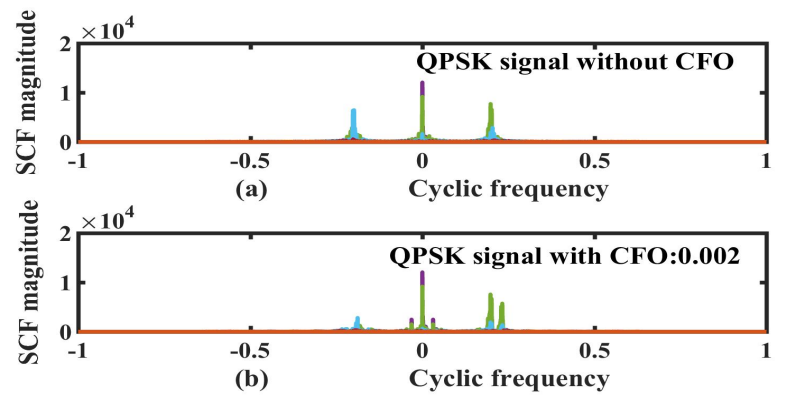

Figure 1: The Effects of Receiver Offsets on the location of the QPSK signal. (a) SCF of QPSK signal without CFO. (b) SCF of QPSK signal with CFO.

The received high frequency wideband signal is first downconverted to an Intermediate Frequency (IF) to the range of Mega-Hertz (MHz) and sampled as a bandpass signal before the correlation for the effective sampling of frequencies. Given a wideband of $N_{B}$ samples divided into $N$ - point slots the relationship can be stated as,

$$
P=\frac{N_{B}}{N}
$$

where $P$ and $N$ are the number of slots and samples per slot respectively. Each slot $P$ is of length $T=N T_{s}$ seconds, where $T_{s}$ is the nominal sampling period. Since we are detecting across the entire wideband, $T_{s}$ is applicable to each slot using a multi-band oscillator. This is different from a wideband approach where $T_{s}$ applies to the entire wideband which makes it difficult for current Analogue to Digital Converters (ADC) to sample or where $T_{s}$ applies to the signals of interest because in real world scenarios, the signals will not be known and therefore makes implementation more complex. Our approach is also different from multi-channel wideband where each channel is sampled with a different oscillator which is not cost effective. The use of a window in the FFT computation reduces spectral leakage as we sample through the wideband. Applying a window to the FFT leads to the computation of the complex envelope in (5) for the $p^{t h}$ slot as,

$$
X(n, f, p)=\sum_{l=-N / 2}^{+N / 2} w(r) x(n-l) e^{-j 2 \pi f(n-l) T_{s}}
$$

where $X(n, f, p)$ is the window-based $w(r)$, narrow-band spectral component of the received signal $x(t)$ for the $p^{t h}$ slot. Considering a multi-slot wideband having the same slot size, the Test Statistic (TS) without the receiver constraints can be expressed as,

$$
\overline{S_{x}^{\alpha}}(n, f)=\frac{1}{P} \sum_{p=1}^{P} S_{x}^{\alpha}(n, f, p)
$$

where $S_{x}^{\alpha}(n, f, p)$ is the discrete SCF for the $p^{t h}$ slot of $N$ samples. This is expressed as,

$$
S_{x}^{\alpha}(n, f, p)=\frac{1}{N} \sum_{n=0}^{N-1} \frac{1}{L} X_{L, p}\left(n, f+\frac{\alpha}{2}\right) X_{L, p}^{*}\left(n, f-\frac{\alpha}{2}\right)
$$

where $X_{L, p}(n, f)$ is the $L$-point window-based FFT of the $p^{t h}$ slot around the $n^{\text {th }}$ sample. Therefore, (11) can be written as,

$$
\begin{aligned}
{\overline{S_{x}^{\alpha}}}^{\alpha}(n, f)=\frac{1}{P} \sum_{p=1}^{P}\left(\frac{1}{N} \sum_{n=0}^{N-1} \frac{1}{L}\right. & X_{L, p}\left(n, f+\frac{\alpha}{2}\right) \\
& \left.\times X_{L, p}^{*}\left(n, f-\frac{\alpha}{2}\right)\right)
\end{aligned}
$$

while $P$ is the number of slots of $N$ size for the wideband over which the SCF is averaged. Equation (13) shows that the Test Statistic (TS) is the correlation of the FFT of the received wideband signal with itself across the wideband ona slot by slot basis as in [18], [19], [20] and [21]. It should be noted that the conventional SCF (6) is a form of the proposed model in (13) where $P=1$ and $N=N^{\prime}$. The cyclic frequency $\alpha$ in (13) is replaced with (7) to represent the presence of CFO and SCO and the offset constrained Test Statistic becomes,

$$
\begin{aligned}
\bar{S}_{x}^{\hat{\alpha}}(n, f)=\frac{1}{P} \sum_{p=1}^{P}\left(\frac{1}{N} \sum_{n=0}^{N-1}\right. & \frac{1}{L} X_{L, p}\left(n, f+\frac{\hat{\alpha}}{2}\right) \\
& \left.\times X_{L, p}^{*}\left(n, f-\frac{\hat{\alpha}}{2}\right)\right)
\end{aligned}
$$

where $T_{s}$ and $T_{s}^{\prime}$ are the sampling period used at the receiver and the actual sampling period with good knowledge of the symbol rate at the transmitter respectively. Also, $\hat{\alpha}$ is the cyclic frequency affected by the CFO and SCO offsets. The second moment or power of the Test Statistics in (13) and (14) can be calculated with,

$$
\left|\bar{S}_{x}^{\alpha}(n, f)\right|^{2} \text { and }\left|\bar{S}_{x}^{\hat{\alpha}}(n, f)\right|^{2}
$$

which are needed to determine the probability of detection shortly. Since the cyclic frequency $\alpha$ is a function of symbol rate $1 / T_{\text {symbol }}$ and signal frequency $f$, the resolution of the cyclic frequency is determined by the FFT size and in a multislot wideband, additionally by the slot size $N$. The cyclic frequency resolution $\Delta_{\alpha}$, of the $N$-point FFT can be expressed as,

$$
\Delta_{\alpha}=\frac{1}{T}=\frac{1}{N T_{s}}=\frac{f_{s}}{N}
$$

where $T, T_{s}, N$ and $f_{s}$ are the sensing period, sampling period, number of samples and sampling rate for the FFTbased slot. The objective is to use the Test Statistics in (14) and its second moment in (15) to get the sizes of FFT and slot that will remedy the effects of the mentioned receiver offsets in the wideband.

\subsection{Threshold and Detection}

A binary decision rule of two hypotheses will be adopted in order to detect the signals.

- Hypothesis $1, H_{1}$ for noise only,

$$
H_{1}: s(t)=w(t)
$$

- Hypothesis $2, H_{2}$ for signal present,

$$
H_{2}: s(t)=x(t)+w(t)
$$

Fundamentally spectral correlation function (SCF) or cyclic autocorrelation function (CAF) is expected to be flat in the presence of noise or exhibits a non-zero mean. From Central limit theorem (CLT) as in [16], [22] and [23], the SCF distribution is Gaussian. The threshold that is approximately 


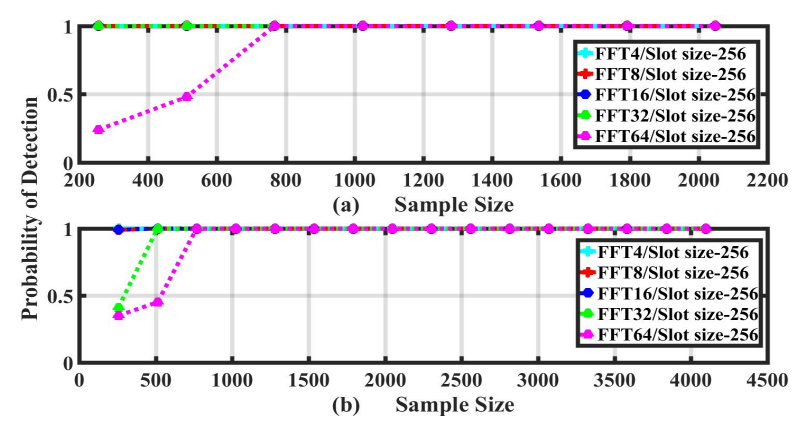

Figure 2: Probability of detection with multi-FFTs at $=5,10$ $\mathrm{MHz}$, Bandwidths $=10,20 \mathrm{MHz}, \mathrm{N}=256$.

at the noise level and gives constant false alarm rate (CFAR) $T_{\mathrm{d}}$ during the detection is selected. The second moment of the Test Statistics (15) at hypotheses 1 and 2 will be represented with $T S_{1}$ and $T S_{2}$ respectively. Both $T S_{1}$ and $T S_{2}$ will be compared against the detection threshold $T_{\mathrm{d}}$ to determine the presence or absence of the signal as,

- $T S_{1}<T_{d}$ signal is absent, $H_{1}$.

- $T S_{2}=>T_{d}$ signal is present, $H_{2}$.

Therefore, the probabilities of detection $P_{\mathrm{d}}$ and false alarm $P_{\text {fa }}$ can be expressed as in (19) and (20) below, If the signal is present, True Positive (TP) is, $T S_{2}=>T_{d}$

$$
P_{d}=\frac{T P}{k k}
$$

where $T S_{2}$ is (15) at signal present, $k k$ and $T P$ represent all the possible positives and the detected true positives respectively. If the signal is absent, False Positive (FP) is, $T S_{1}<T_{d}$

$$
P_{f} a=\frac{F P}{k k}
$$

where $T S_{1}$ is (15) at noise only, $k k$ and $F P$ represent all the possible negatives and the detected false positives respectively.

\section{Results and Discussion}

\subsection{The Effect of Bandwidth and Slot Size N and Number of $\mathbf{P}$}

Let us look at the performance of the Hanning window-based FFTs with respect to the size of the bandwidth. For the purpose of correlation, the FFTs must be in the powers of 2 . Given a bandwidth $B \mathrm{MHz}$, sampling rate $f_{s}=2 B$ and assuming fixed $\mathrm{CFO}=\mathrm{SCO}$ at $1 \times 10^{-} 3$ and fixed sensing period $T$ of $2 \times 10^{-4}$ seconds for the wideband. Figs. $2 \mathrm{a}$ and $2 \mathrm{~b}$ show the detection performance for two different bandwidths $B$ of 10 and $20 \mathrm{MHz}$ respectively. According to (9) the corresponding total samples is $N_{B}$. In both Figs. $2 \mathrm{a}$ and $2 \mathrm{~b}$, given a fixed slot size $N$ of 256 samples, the expected ideal performance for an individual FFT is having the peak probability of 1 with a small number of samples as possible to achieve the objective of finding a low-complexity and effective detection model. FFT 4, 8, 16 and 32 present better probability of detection (Pd) in Fig. 2a with FFT 32 requiring more samples alongside FFT 64 in Fig. 2b for larger bandwidths. In Fig. 3b and for the same bandwidth and sample size (4500) as in Fig. $2 b$ but with larger $N$ of 512 samples, FFT 32 shows similar Pd with FFTs 4,8 and 16 due to the increased slot size. Therefore, the smaller slots will produce less total resultant samples than the larger ones. Since bandwidth is directly proportional to total number of samples (9), the results in Figs 2 and 3 show that the Pd for an FFT in the correlation (15) is a function of



Figure 3: Probability of detection with multi-FFTs at $\mathrm{f}=5,10$ $\mathrm{MHz}$, Bandwidths $=10,20 \mathrm{MHz}$ and $\mathrm{N}=512$.

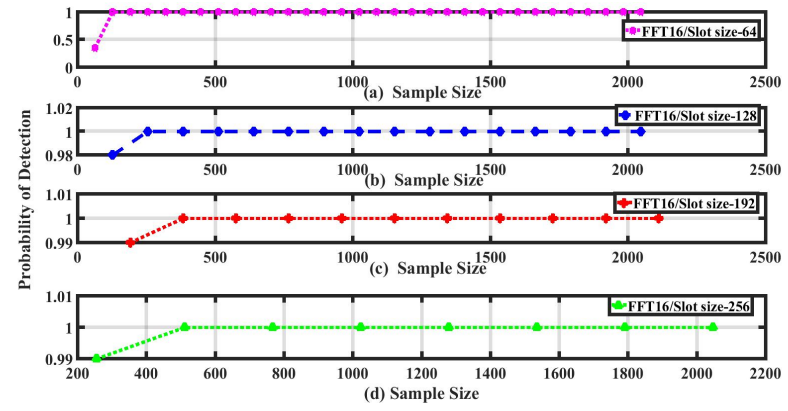

Figure 4: Probability of detection with fixed FFT 16 and multislot sizes at $\mathrm{f}=5 \mathrm{MHz}$, Bandwidths $=10 \mathrm{MHz}$.

both the bandwidth and the slot size. For the same slot size and different bandwidths, the performance of the FFTs defer concerning the number of samples for peak detection which implies that the bandwidth affects the choice of slot $\operatorname{size} N$ and from (9), the choice of numbers of slots $P$.

\subsection{Optimizing the Slot Size $\mathbf{N}$ and Number $P$}

As stated earlier, from (9), the number of slots $P$ is affected by the bandwidth $N_{B}$ or slot size $N$. Therefore, there is need to optimize $N$ and $P$ for maximum $P_{d}$ for a given bandwidth. Also there is additional constraint to $N$ in order to cover the minimum samples $N$ min required for information symbols within one slot. The optimization problem can be formulated from (9) and (19) as,

$$
(\bar{N}, \bar{P})=\underset{N, P}{\operatorname{argmin}} P_{d}
$$

such that $N P=N_{B}$ and $N \leq N_{\text {min }}$, where $\bar{N}$ and $\bar{P}$ are the optimized $N$ and $P$ and $P_{d}$ is the probability of detection. It is shown in Fig. 4, that with smaller $N$ less samples are required for peak detection across different sizes of $N$ for a fixed bandwidth of $10 \mathrm{MHz}$, fixed sensing period $T$ of $2 \times$ $10^{-4}$ seconds. This will also result in an increase in $P$ as in (9). There will be an increase in $P$ if $N$ is further reduced and will subsequently produce an increase in the number of FFTs which could impact on the computational complexity which is a constraint. In order to further solve the optimization problem in (21), consideration will be given to the overall complexity from combining multiple FFTs; which, for a single FFT complexity is $L \log _{2} L$.

\subsection{Computational Complexity}

Given the computational complexity $F F T_{c x}$ for 1 slot as,

$$
F F T_{c x}=M\left(L \log _{2} L\right)
$$

where $L$ is the window-based FFT size and $M$ is the total number of FFTs required for 1 slot and expressed as,

$$
M=\frac{N}{L}
$$




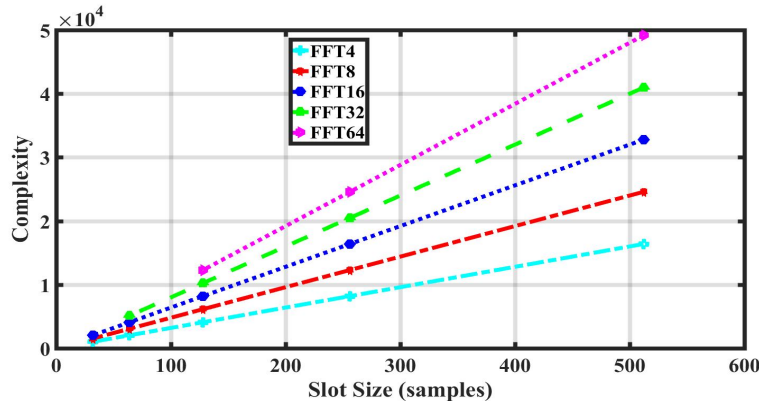

Figure 5: Complexity for different slot sizes and FFTs.

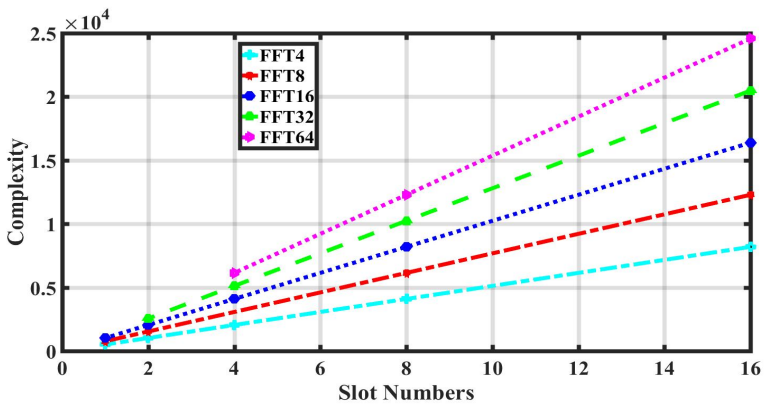

Figure 6: Complexity for different slot numbers and FFTs.

From (9), (22) and (23), we derive the total computational complexity $F F T_{t c}$ for the wideband as,

$$
F F T_{t c}=P \times M \times L \log _{2} L
$$

The smaller $N$ will generate more slots $P$ and consequently more $M$ depending on $L$. We will obtain the optimum $L$ and $M$ from the second optimization problem in (25) to further solve (21) within the constraints of computational complexity. Additional constraint should be added to in order to cover the minimum samples required $L_{\text {min }}$ for the information in each slot.

$$
(\bar{L}, \bar{M})=\underset{L, M}{\operatorname{argmin}} P_{d}
$$

such that $L M \leq N$ and $L \geq L_{\min }$ where $\bar{L}$ and $\bar{M}$ are the optimized $L$ and $M$. From (9), (23) and (24) we can verify the effect of computational complexity. In Fig. 5, the smaller $N$ produces lower complexity but on the whole FFT length 4 shows better complexity for the wideband $F F T_{t c}$ (24) even at increasing slot size $N$. The lower complexity performance using FFT4 is also supported in Fig. 6 at $N$ of 256 samples in addition to increasing $P$. From Figs 5 and 6, it is shown that the use of small FFTs for the correlation in (15) does not impact the model in terms of complexity as well as the smaller $N$. Applying this condition to the first optimization (21), subject to a given bandwidth, $N$ and $P$ can be chosen as low as computationally possible to maximize the detection performance. From (23), it is understood that the smaller FFTs will result in an increased number of FFTs $M$ for any given bandwidth. Although there is increased number of FFTs using the smaller FFTs with sizes 4, 8 and 16 in Fig. 7, the total complexity due to a small FFT is small compared with the larger FFTs. Applying this to the optimization problem in (25), means that the increase in $M$ is not a disadvantage as long as $L$ is small. Therefore, the use of small $N$ and $L$ satisfies the optimization requirements in (21) and (25) and the comparative minimum complexity constraints in (25). The performance of the Test Statistic in terms of Complexity can be compared against the conventional DFT. From calculations of DFT complexity which is $N^{2}$ for different slot and FFT sizes, the gain in complexity of the Test Statistic over the



Figure 7: Complexity for increasing number of FFTs.

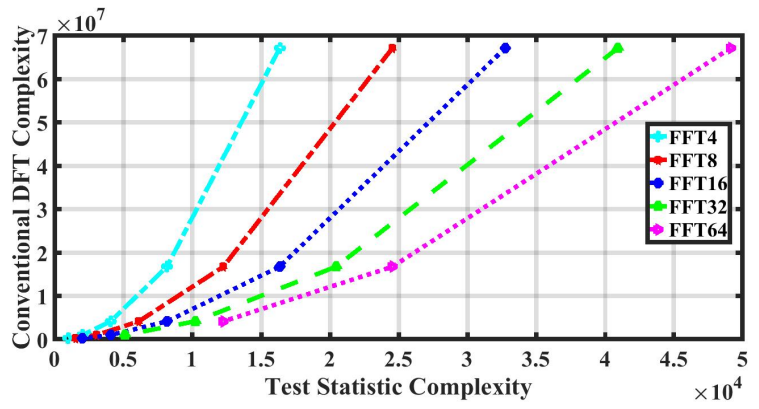

Figure 8: Comparison between Model Statistic and Conventional DFT Complexities.

conventional DFT increases more with the small FFTs as in Fig. 8. The smaller the FFT size the more the difference in the complexities between the Conventional DFT and Test Statistic.

\subsection{The use of small FFTs}

Considering all the results and calculations, the smaller the FFT, the better is the detection performance across small and big slot and total sample sizes. Although there is an increased number of FFTs using the small FFTs and small slot sizes, the result that they still offer comparative lower complexities than the larger ones, makes it acceptable to adopt them in the model. From (6), (14) and (15), the sum product of the Test Statistic is a function of FFT and slot sizes and number $L, N$ and $P$ respectively. Therefore for the small FFTs to function across wider bandwidths depends on the $N$ and $P$ with larger bandwidths requiring larger $N P$. Previously, it has been established that the smaller $N$ will produce more $P$ and recalling that the small FFTs still offer comparative lower complexities across increasing $P$ as in Fig. 6, therefore they are applicable to other higher bandwidths.

\subsection{Applying the Optimization}

If the number of slots $P=1$, the product of correlating the FFTs in $(15)=$ and gives the maximum computable $P$ of 256. According to the optimization requirements in (21) and (25), selecting $L$ as FFT16 and minimizing $N$ as 64 gives $64 P s$. Given a bandwidth $N_{B}$ of 4096 samples, from (9), $N=N_{B} / P=4096 / 64=64$ samples. From (23), the number of FFTs $M=64 / 4=16$ FFTs. In summary, considering the simulations and calculations, given a bandwidth $N_{B}$ of 4096 samples, the optimized values of $L, M, N, P$ are 16, 16, 64 and 64 respectively. Therefore, we can obtain optimized values of $M, N, P$ for a selected small $L$. These values are then used in comparing the performance of the model with and without receiver constraints at different values of SNR as in Fig. 9. There is a closeness between a pair of curves for both with and without offsets obtained for each SNR. This shows that the effects of receiver offsets have been reduced 


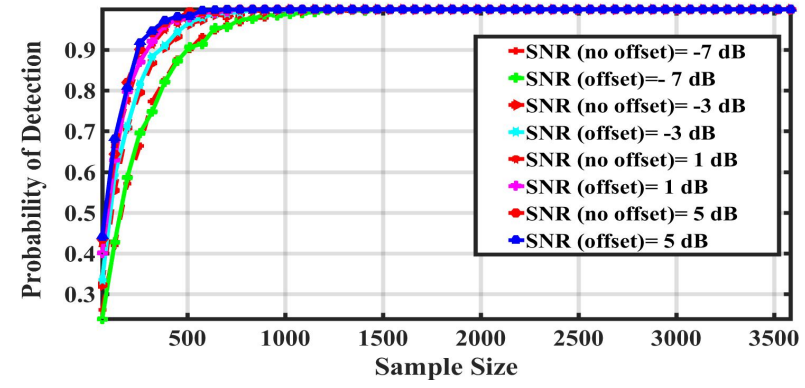

Figure 9: Probability of detection with optimized Slot/FFT of $64 / 16$.

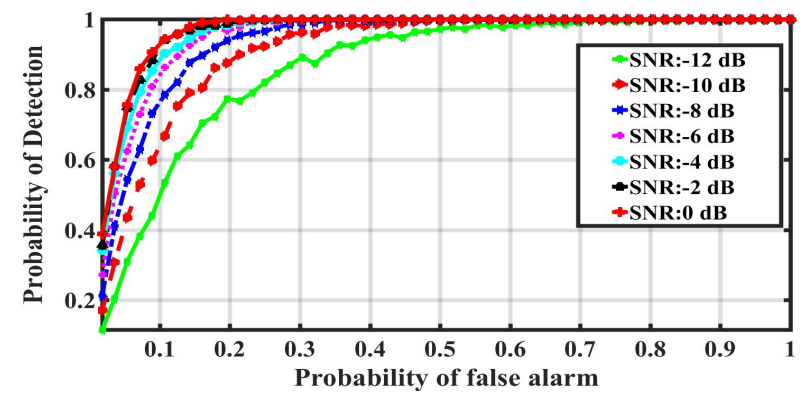

Figure 10: Receiver Operating Characteristic at $2.5 \mathrm{MHz}$ QPSK signal, slot-64/FFT-16.

by adopting small FFT and slot sizes. It also reduces the concern that large samples will be needed for resampling for cyclostationary feature detection under receiver impairments. Fig. 10 is the receiver operating characteristic (ROC) with the slot/FFT size pair $(64,16)$ at different low signal levels with SCO and CFO. Fig. 10 shows the detection performance of the wideband cyclostationary feature detector with receiver constraints. In the presence of receiver constraints, low signal levels down to $-12 \mathrm{~dB}$ SNR are still detectable by the Test Statistic in (14). The proposed model detects much lower level signals under receiver constraints as shown in Figs. 9 and 10 when compared against a time-based model discussed in [16]. It offers lower complexity, speed and accuracy due to the small size FFTs.

\section{CONCLUSION}

In this paper, we have shown that the effects of sampling clock and cyclic frequency offsets can be reduced by using the optimized sizes and numbers of slot size and fast Fourier transform for the implementation. It has been shown that the use of multi-slot and small FFTs do not increase the computational complexity of the model. This is in contrast to the approach of using large samples considering the cost and computational complexity. This model serves as a guide to the hardware design and implementation of wideband cyclostationary feature detectors under receiver constraints.

\section{References}

[1] K. Patil, R. Prasad, and K. Skouby, "A survey of worldwide spectrum occupancy measurement campaigns for cognitive radio," in International conf. on devices and communications (ICDeCom), 2011, pp. 1-5.

[2] P. Kolodzy and et al., "Spectrum policy task force report," Federal Communications Commission,, Washington, USA, ET Docket . 02-135, 2002

[3] D. Tarchi and et al, "Technical challenges for cognitive radio application in satellite communications," in IEEE 9th International conf. on CR oriented wireless networks, Oulu, Finland, Jun. 2014, pp. 136-142.
[4] M. Subhedar and G. Birajdar, "Spectrum sensing techniques in cognitive radio networks: A survey," Iinternational journal of next-generation networks (IJNGN), vol. 3, pp. 37-51, Jun. 2011.

[5] T. Yucek and H. Arslan, "A survey of spectrum sensing algorithms for cognitive radio applications," IEEE communications survey \& tutorials, vol. 11, pp. 116-130, Mar. 2009.

[6] D. Cabric, S. M. Mishra, and R. W. Brodersen, "Implementation issues in spectrum sensing for cognitive radios," in IEEE 38th Asilomar Conf. Signals, Systems and Computers, Pacific Grove, USA, Mar. 2004, pp. 772-776.

[7] S. Ren, Z. Zeng, C. Guo, and X. Sun, "Wideband spectrum sensing based on coprime sampling,", in IEEE 22nd International conf. on telecommunications (ICT), Sydney, Australia, Mar. 2015, pp. 248-352.

[8] D. M. Najafabadi, A. A. Tadaion, and M. R. A. Sahaf, "Wideband spectrum sensing by compressed measurements," in IEEE Computers and Communications Symposium, Cappadocia, Turkey, Jul. 2012, pp. 667-671.

[9] W. A. Gardner, Introduction to random processes with applications to signals and systems. New York: McGraw-Hill, Inc, 1990.

[10] K. Kim and et al, "Cyclostationary approaches to signal detection and classification in cognitive radio," in IEEE New frontiers in dynamic spectrum access networks (DySPAN), Dublin, Apr. 2007, pp. 212-215.

[11] Y. Zeng and Y. Liang, "Robustness of the cyclostationary detection to cyclic frequency mismatch," in IEEE 21st International symposium on personal indoor and mobile radio communications (PIMRC), Istanbul, Sep. 2010, pp. 2704-2709.

[12] W. A. Gardner, "Signal interception: unifying theoretical framework for feature detection," IEEE Transactions on communications, vol. 36, No. 8, pp. 897-906, Aug. 1988.

[13] A. Sahai and D. Cabric, "Cyclostationary feature detection," in IEEE Conf. on dynamic spectrum access network (DySPAN), California, USA, 2005, pp. 1-69.

[14] A. V. Dandawate and G. B. Giannakis, "Statistical tests for presence of cyclostationarity," IEEE Transactions on signal processing, vol. 42, No. 9, pp. 2355-2369, Sep. 1994.

[15] E. Rebeiz, V. Jain, and D. Cabric, "Cyclostationary-based low complexity wideband spectrum sensing using compressive sampling," in IEEE ICC CRo and networks symposium, Ottawa, ON, Jun. 2012, pp. 1619-1623.

[16] E. Rebeiz, P. Urriza, and D. Cabric, "Optimizing wideband cyclostationary spectrum sensing under receiver impairments," IEEE Transactions on signal processing, vol. 61, No. 15, pp. 3931-3943, May 2013.

[17] R. S. Roberts, W. A. Brown, and H. H. Loomis, "Computationally efficient algorithms for cyclic spectral analysis," IEEE signal Processing Society, vol. 8, No. 2, pp. 28-49, Apr. 1991.

[18] K. Po and J. Takada, "Signal detection based on cyclic spectrum estimation for cognitive radio in ieee 802.22 wran system," The institute of electronics, information and communication (IEICE), pp. 1-5, 2007.

[19] D. Yoo, J. Lim, and M. Kang, "Ieee atsc digital television signal detection with spectral correlation density, communications and networks," Communications and networks, vol. 16, No. 6, pp. 600-612, Dec. 2014.

[20] H. Chen, W. Gao, and D. G. Daut, "Spectrum sensing using cyclostationary properties and application to ieee 802.22 wran," in IEEE GLOBECOM, Washington, DC, Dec. 2007, pp. 31333138.

[21] Y. Wen-jing, Z. Bao-yu, and M. Qung-min, "Cyclostationary property based spectrum sensing algorithms for primary detection in cognitive radio systems," Springer, pp. 676-680, Dec. 2009.

[22] M. Derakhshani, M. Nasiri-Kenari, and T. Le-Ngoc, "Cooperative cyclostationary spectrum sensing in cognitive radios at low snr regimes," in IEEE International conf. on communications, Capetown, South Africa, May 2010, pp. 1-5.

[23] R. T. Short, "Computation of rice and noncentral chisquared probabilities," Phaselocked Systems, Technical Report . PHSO254, 2012. 